ćclaircies. Note technique, $\mathrm{n}^{\circ} .24$ de la division asylviculturen. - Decourt, N.; Bachacou, J., 1976: Erude de la compétition dans des plantations régulières par. la méthode des variables régionalisées. Annales des sciences forestières. Fasc. 4, p. 177-198. - DelvauX, J., 1975: Acquisition du rang social dans les jeunes plantations d'épicća - série $\mathrm{B}-\mathrm{n}^{\circ} 39$ des travaux de la stazion de recherches des eaux ex forêts de Groenendaal-Hoeilaart. - Ders., 1968: L'ćclaircie, définition er points de vue. mars 1968 - document stencilé. - Ders., 1964: A propos de l'éclaircie des hêtraies en forêt de Soignes: les aspects qualitarifs. Travaux série $B-n^{\circ} 30$ de la station de recherches des Eaux et Forêts de Groenendaal-Hoeilart. Ducellter, U., 1930: La forêt de Bellême et une nouvelle méthode d'éclaircies. Revue des Eaux et Forêts, $N^{\circ} .4$, p. 263-278. - FraNZ, F., 1971: Grundlagen und Verfahren standortbezogener Leistungsschätzung. Forschungsberichte FFA München, Nr. 2. - Ders., 1974: Zur Fortentwicklung der Durchforstungsverfahren aus der Sicht der Waldertragskunde. Forstarchiv, H. 2/3, S. 28-34. - HufFEL, G., 1929: Les débuts de l'enseignement forestier en France. Annales de l'Ecole nationale des Eaux et Forêts, tome III, fasc. 1, p. 3-135. - Ilty, G.; Lemoine, B., 1970: Densité de peuplement, concurrence et coopération chez le pin maritime. Annales des sciences forestières, no.2, p. 127-155. - KeNNEl, R., 1972: Die Buchendurchforstungsversuche in Bayern von 1970 bis 1970 . Forschungsberichte der Forstliden Forschungsanstalt München, Nr. 7. - KRAMER, H., 1977; Aspekte der Durchforstung: Weltweit gesehen. Allg. Forstz., Nr.16. - LeibundGuT, H., 1976: Grundlagen zur Jungwaldpflege - Mitteilungen der eidgenössischen Anstalt für das forstliche Versuchswesen. 52, 313-371. - Nelder, J. A., 1962: New kinds of systematic designs for spacing experiments. Biometrics 18, 283-307. - Oswald, H., 1977: Expérience européenne de traitement de l'épicéa: premiers résultats du dispositif expérimental français in "Der europäische Stammzahlversuch in Fichte". Mitteilungen der forstlichen Versuchs- und Forschungsanstalt BadenWürttemberg, H. 80, S. 55-59; complété par une note (Sept. 1977) concernant la deuxième éclaircie. - Oswald, H.; PardE, J., 1976: Une expérience d'espacement de plantation de Douglas en forêt domaniale d'Amance. Revue forestière française, $\mathrm{n}^{\circ} .3$, p. 185-192. Otrorint, J. M., 1977: L'étude quantitative du développement et de la croissance des peuplements forestiers équiennes purs. Document 77/01 à diffusion limitée. - Pardé, J., 1972: Das forstliche Versuctswesen in Frankreich. Beziehungen zu den Nachbarstaaten. Verleihung der Wilhelm-Leopold-Pfeil-Preise 1972, Freiburg. - Ders., 1977: Les relations

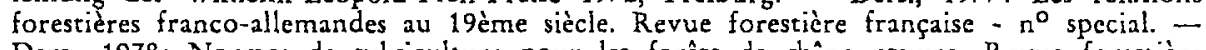
Ders., 1978: Normes de sylviculture pour les forêts de chêne rouvre. Revue forestic̀re française, $\mathrm{n}^{0}$. - SchовER, R., 1972: Die Rorbuche. J. D. Sauerländer's Verlag. - vaN Miegroet, M., 1967: Enquête sur l'ćclaircie. Sylva Gandavensis, nº. 2.

Anschrift des Verfassers: Prof. J. PARDE, I.N.R.A. - C.N.R.F., Station de Sylviculture et de Production Champenoux, F-54280 Seichamps

\title{
MITTEILUNG
}

\section{Forstliche Hochschulwoche 1979}

In der Zeit yom 9. bis 11. Oktober 1979 findet in Freiburg die Forstliche Hochschulwoche 1979 statt.

Die Programme werden im Dekanat der Forstwissenschaftlichen Fakultät der Universitär Freiburg (Erbprinzenstraße 13) voraussichtlich ab Mitte Mai 1979 vorliegen; Anmeldungen zur Hochschulwoche sollten beim Dekanat nach Möglichkeit bis 1. September 1979 eingehen. 